Old Dominion University ODU Digital Commons

1991

\title{
Parametric Instability of Supersonic Shear Layers Induced by Periodic Mach Waves
}

Fang Q.Hu

Old Dominion University, fhu@odu.edu

Christopher K. W. Tam

Follow this and additional works at: https://digitalcommons.odu.edu/mathstat_fac_pubs

Part of the Applied Mathematics Commons, Fluid Dynamics Commons, and the Plasma and Beam Physics Commons

\section{Repository Citation}

Hu, Fang Q. and Tam, Christopher K. W., "Parametric Instability of Supersonic Shear Layers Induced by Periodic Mach Waves" (1991). Mathematics \& Statistics Faculty Publications. 26.

https://digitalcommons.odu.edu/mathstat_fac_pubs/26

\section{Original Publication Citation}

Hu, F. Q. \& Tam, C. K. W. (1991). Parametric instability of supersonic shear layers induced by periodic mach waves. Physics of Fluids A: Fluid Dynamics, 3(6), 1645-1656. doi:10.1063/1.857943

This Article is brought to you for free and open access by the Mathematics \& Statistics at ODU Digital Commons. It has been accepted for inclusion in Mathematics \& Statistics Faculty Publications by an authorized administrator of ODU Digital Commons. For more information, please contact digitalcommons@odu.edu. 
Parametric instability of supersonic shear layers induced by periodic Mach waves

Fang Q. Hu, and Christopher K. W. Tam

Citation: Physics of Fluids A: Fluid Dynamics 3, 1645 (1991); doi: 10.1063/1.857943

View online: http://dx.doi.org/10.1063/1.857943

View Table of Contents: http://aip.scitation.org/toc/pfa/3/6

Published by the American Institute of Physics 


\title{
Parametric instability of supersonic shear layers induced by periodic Mach waves
}

\author{
Fang Q. Hu \\ Department of Mathematics and Statistics, Old Dominion University, Norfolk, Virginia 23529 \\ Christopher K. W. Tam \\ Department of Mathematics, Florida State University, Tallahassee, Floridä 32306-3027
}

(Received 27 June 1990; accepted 7 February 1991)

\begin{abstract}
It is suggested that parametric instability can be induced in a confined supersonic shear layer by the use of a periodic Mach wave system generated by a wavy wall. The existence of such an instability solution is demonstrated computationally by solving the Floquet system of equations. The solution is constructed by means of a Fourier-Chebyshev expansion. Numerical convergence is assured by using a very large number of Fourier and Chebyshev basis functions. The computed growth rate of the induced flow instability is found to vary linearly with the amplitude of the mach waves when the amplitude is not excessively large. This ensures that the instability is, indeed, tied to the presence of the Mach waves. It is proposed that enhanced mixing of supersonic shear layers may be achieved by the use of such a periodic Mach wave system through the inducement of parametric instabilities in the flow.
\end{abstract}

\section{INTRODUCTION}

Recent experiments on high-speed mixing layers by Ikawa and Kubota, ${ }^{1}$ Papamoschou and Roshko, ${ }^{2,3}$ and Chinzei et al. ${ }^{4}$ revealed that the mixing or spreading rate of these flows decreased rapidly as Mach number became supersonic. In addition, Papamoschou and Roshko found that the normalized mixing rate as a function of convective mach number correlated extremely well with the normalized maximum growth rate of the instability waves of these flows. This strongly suggests that flow instability is responsible for the mixing of high-speed shear layers. Further, the decrease in mixing rate with increase in Mach number is due to the fact that, at higher Mach numbers, the instability waves of these flows have a much reduced spatial growth rate. Since the pioneering work of Papamoschou and Roshko, a number of papers have appeared aiming to improve the calculated growth rates of the flow instabilities and their correlation with the measured spreading rate. This includes the use of the spatial rather than the temporal growth rates. ${ }^{5-8}$ The importance of wall reflections is pointed out ${ }^{9-11}$ and the inclusion of the wall effects further improves the agreement between the calculated results and measurements. ${ }^{12}$ It is now generally accepted that flow instability is, indeed, the primary mechanism of fluid mixing in supersonic shear layers. To enhance mixing, an obvious nccessity is to enhance flow instabilities.

The purpose of this paper is to draw attention to the possibility of enhancing flow instability of confined supersonic mixing layers by the use of a periodic Mach wave system generated by a wavy wall as shown in Fig. 1. An advantage of this scheme is that no intrusive objects need be placed inside the flow. Such objects would inevitably produce shock waves and dissipation. A periodic Mach wave system can induce flow instability in two ways. First, is through resonant instability ${ }^{13,14}$ by - which two acoustic modes of the ducted shear layer flow are driven into resonance and insta- bility. It is possible to show that, by choosing properly the wavelength of the Mach waves, the interaction of one acoustic wave mode with the Mach wave system produces a forcing on the other mode and vice versa. In this way, the two acoustic modes force each other simultaneously leading to growth and instability. Second, is through parametric instability, which is akin to the secondary instabilities of lowspeed boundary layers and shear layers studied extensively by Herbert ${ }^{15}$ and Orszag and Patera. ${ }^{16}$ In the presence of a periodic Mach wave system, the mean flow is spatially periodic in the flow direction. This periodic mean flow can support new parametric instability waves. It is the objective of this paper to show this computationally. For this purpose, consideration will be confined to temporal instability only. In order to demonstrate that the presence of a periodic Mach wave system can induce new instability waves, it is necessary to show such waves exist. This is done here by expanding the instability wave solution according to Floquet theory in a Fourier series in the flow direction and in Chebyshev polynomials in the transverse direction. The number of Fourier and Chebyshev terms are increased until satisfactory numerical convergence is assured. Furthermore, it will be shown that the growth rate of the new instability wave depends linearly on the amplitude of the Mach wave system when the amplitude is not very large. This ensures that the new instability wave is, indeed, tied to the presence of the periodic Mach waves.

It is worthwhile to point out that although the parametric instability analyzed here is in many ways similar to the secondary instabilities of subsonic boundary layers, there are yet major differences. First of all, the boundary-layer secondary instabilities are induced by the vorticity of the primary Tollmien-Schlichting waves. In the case of supersonic shear layers penetrated by periodic Mach waves, the mechanism of parametric instability is not completely clear at this time. Most likely it is driven by pressure or acoustic resonance. In any case, vorticity plays very little or no role at all. 


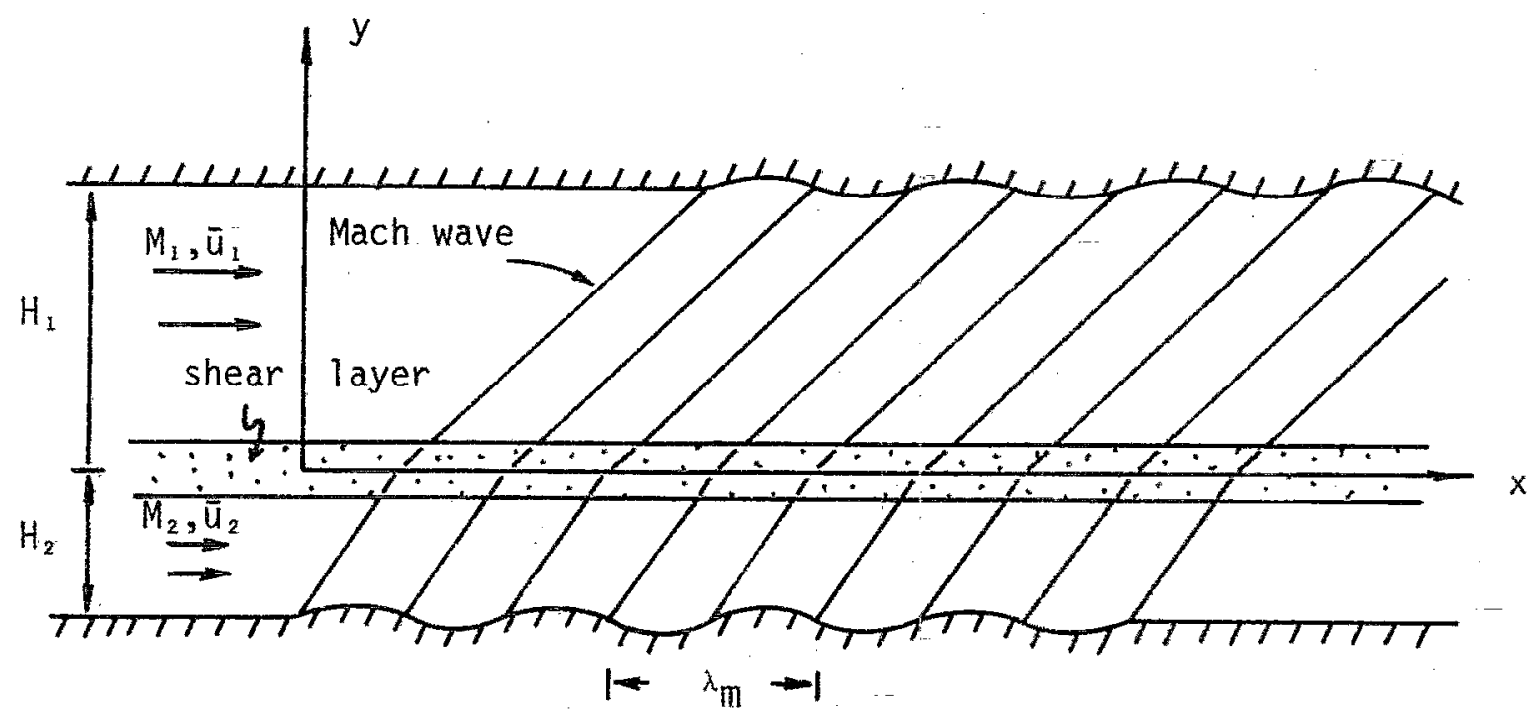

FIG. 1. Schematic diagram showing a periodic Mach wave system generated by wavy walls superimposing on a supersonic shear layer.

In Iferbert's analysis of the secondary instabilities, only one or two Fourier terms are all that is necessary to provide an adequate instability wave solution. In the present case, however, a much larger number of Fourier terms are needed to insure convergence in the eigenvalue and eigenfunction. Twenty-eight Fourier terms are retained in the example to be discussed in Sec. V.

The scope of the present study is limited. It is focussed exclusively on the existence of parametric or Floquet instability waves in a supersonic shear layer induced by the presence of a periodic Mach wave system. No parametric study of the most unstable configuration will be carried out.

\section{PERIODIC MACH WAVES}

Consider a supersonic shear layer confined within two wavy parallel walls as shown in Fig. 1. It will be assumed that the Mach waves are generated by the lower wall. Once generated, the Mach waves propagate up passing through the shear layer and eventually reach the top wall. For simplicity, the amplitude and phase of the top wavy wall will be adjusted so that no reflection will occur. That is, no reflected wave will emanate from the top to the bottom wall. It is recognized that this is not the most practical Mach wave configuration for applications where the flow conditions may vary. However, it can be implemented for a single shear flow (design) condition. Here, this nonrefiecting upper wall condition is chosen strictly for the purpose of simplifying the analysis:

Let the equations of the wavy walls be,

$$
\begin{array}{ll}
y=-H_{2}+A_{0} \sin \left(k_{m} x\right) & \text { (bottom wall), } \\
y=H_{1}+B_{0} \sin \left(k_{m}+\phi\right) & \text { (top wall). }
\end{array}
$$

The strength of the Mach waves depends on the ratio of the amplitude $A_{0}$ of the bottom wavy wall to the wavelength $\lambda_{m}\left(=2 \pi / k_{m}\right) .{ }^{17}$ This dimensionless ratio will be denoted by $\epsilon$, i.e.,

$$
\epsilon=A_{0} / \lambda_{m}=A_{0} k_{m} / 2 \pi
$$

Throughout this work, $\epsilon$ will be taken to be no more than $2 \%$. For small $\epsilon$, a linear Mach wave solution will suffice.

Now suppose the shear layer is confined within the rcgion $-\delta \leqslant y \leqslant \delta$. Outside this region the flow is uniform so that the pressure perturbation associated with the Mach waves can readily be found ${ }^{17}$ as

$p_{m}:=\left\{\begin{array}{c}{\left[\bar{\rho}_{1} \bar{u}_{1}^{2} 2 \pi \epsilon \alpha /\left(M_{1}^{2}-1\right)^{1 / 2}\right] \cos \left[k_{m} x-k_{m}\left(M_{1}^{2}-1\right)^{1 / 2}\left(y-H_{1}\right)+\phi\right], \quad \delta<y \leqslant H_{1},} \\ {\left[\bar{\rho}_{2} \bar{u}_{2}^{2} 2 \pi \epsilon /\left(M_{2}^{2}-1\right)^{1 / 2}\right] \cos \left[k_{m} x-k_{m}\left(M_{2}^{2}-1\right)^{1 / 2}\left(y+H_{2}\right)\right]} \\ \quad+C \cos \left[k_{m} x+k_{m}\left(M_{2}^{2}-1\right)^{1 / 2} y+\beta\right], \quad-H_{2}<y<-\delta,\end{array}\right.$

where $M_{1}$ and $M_{2}$ are the Mach numbers on the two sides of the shear layer. The second term in the region $-H_{2} \leqslant y \leqslant-\delta$ represents a weak reflected wave off the shear layer with amplitude $C$ and phase $\beta$. Here, $C$ is nu merically small but is retained in the stability analysis. The reflected wave will again be reflected off the bottom wall. This secondary reffected wave will be neglected. The corresponding velocity perturbations of the Mach waves are

$$
\begin{aligned}
& u_{m}=-\left(\bar{\rho}_{i} \bar{u}_{i}\right)^{-1} p_{m}, \\
& v_{m}=\left(M_{i}^{2}-1\right)^{1 / 2}\left(\bar{\rho}_{i} \bar{u}_{i}\right)^{-1} p_{m},
\end{aligned}
$$

where subscript $i$ in the above expression is equal to 1 for $\delta \leqslant y \leqslant H_{1}$ and 2 for $-H_{2} \leqslant y \leqslant-\delta$.

Inside the shear layer, the pressure associated with the Mach waves is governed by the time-independent linearized equation of motion for a compressible flow. Since the solu- 
tion is periodic in $x$, it can be represented in the form

$$
p_{m}=\operatorname{Re}\left[f(y) e^{i k_{m} x}\right], \quad-\delta<y \leqslant \delta,
$$

where $\operatorname{Re}[\mathrm{]}=$ the real part of Eq. (2). From the governing equation for $p_{m}$, it is easily found that the function $f(y)$ must satisfy the equation

$$
\frac{d^{2} f}{d y^{2}}-\left(\frac{2}{\bar{u}} \frac{d \bar{u}}{d y}+\frac{1}{\bar{\rho}} \frac{d \bar{\rho}}{d y}\right) \frac{d f}{d y}+\left(\frac{\bar{u}^{2}}{\bar{a}^{2}}-1\right) k_{m}^{2} f=0
$$

where $\bar{u}(y), \bar{\rho}(y)$, and $\bar{u}(y)$ are the mean velocity, density, and speed of sound distribution in the channel. In this work, $\bar{u}$ will be approximated by the hyperbolic tangent velocity profile used in Ref. 10, i.e., $\bar{u}=0.5\left[\bar{u}_{1}+\bar{u}_{2}+\left(\bar{u}_{1}-\bar{u}_{2}\right)\right.$ $\times \tanh \left(2 y / \delta_{\omega}\right)$ ], where $\delta_{\omega}$ is the vorticity thickness of the shear layer. The mean density and speed of sound are calculated by Crocco's relation ${ }^{17}$ and constant static pressure. The expressions of Eq. (1) may be casted in the same form as (2) by replacing the cosine function by its equivalent in the form of the sum of two exponential functions. They provide the initial conditions for integrating (3). With these initial conditions, ( 3 ) may be integrated numerically from $y=\delta$ to $y=0$ and from $y=-\delta$ to $y=0$. The requirements of continuity of $f$ and its derivative at $y=0$ then determine the four unknowns $\alpha, \phi, C$, and $\beta$. In this way, the periodic Mach wave field is calculated. For the case $M_{1}=3.5, M_{2}=1.2$, $H_{1}=H_{2}=0.5 H, \delta_{\omega}=0.1 H, \lambda_{m}=0.38 H$, and equal total temperature in the two streams, the numerical values of these parameters are found to be $\alpha=0.766, \phi=1.29$, $\beta=0.475$, and $C=0.0115 \epsilon \bar{\rho}_{1} \bar{u}_{1}^{2}$. Under these conditions the pressure amplitude, $2 \pi \bar{\rho}_{2} \bar{u}_{2}^{2} \epsilon /\left(M_{2}^{2}-1\right)^{1 / 2}$, associated with the Mach waves incident on the shear layer [see Eq. (1)] is equal to $1.11 \bar{\rho}_{1} \bar{u}_{1}^{2} \epsilon$. This is much larger than the amplitude of the waves reflected off the shear layer.

\section{FORMULATION}

Now consider small amplitude disturbances superimposed on the mean flow and the periodic Mach waves. The flow variables may be decomposed into three parts as,

$$
\begin{aligned}
& {\left[\begin{array}{c}
u \\
v \\
w \\
p
\end{array}\right]=} {\left[\begin{array}{c}
\bar{u} \\
0 \\
0 \\
\bar{p}
\end{array}\right]+\epsilon\left[\begin{array}{c}
\hat{u}_{m} \\
\hat{v}_{m} \\
0 \\
\hat{p}_{m}
\end{array}\right]+\left[\begin{array}{c}
u^{\prime} \\
v^{\prime} \\
w^{\prime} \\
p^{\prime}
\end{array}\right] } \\
& \equiv[\text { mean flow }]+[\text { Mach waves }] \\
&+[\text { perturbation }] .
\end{aligned}
$$

The disturbance variables must satisfy the linearized equations of motion. On including the effect of viscosity and heat conductivity, the momentum and energy equations are

$$
\begin{aligned}
\frac{\partial u^{\prime}}{\partial t}+ & \left(\bar{u}+\epsilon \hat{u}_{m}\right) \frac{\partial u^{\prime}}{\partial x}+\epsilon \hat{v}_{m} \frac{\partial u^{\prime}}{\partial y}+\left(\frac{1}{\bar{\rho}}-\frac{\epsilon \hat{p}_{m}}{\bar{\rho}^{2}}\right) \frac{\partial p^{\prime}}{\partial x} \\
& -\mu \frac{1}{\bar{\rho}}\left(\frac{\partial^{2} u^{\prime}}{\partial x^{2}}+\frac{\partial^{2} u^{\prime}}{\partial y^{2}}+\frac{\partial^{2} u^{\prime}}{\partial z^{2}}\right) \\
& +\epsilon \frac{\partial \hat{u}_{m}}{\partial x} u^{\prime}+\left(\frac{d \bar{u}}{\partial y}+\epsilon \frac{\partial \hat{u}_{m}}{\partial y}\right) v^{\prime} \\
& -\frac{\epsilon}{\gamma \bar{p} \bar{p}} \frac{\partial \hat{p}_{m}}{\partial x} p^{\prime}=0 \\
\frac{\partial v^{\prime}}{\partial t}+ & \left(\bar{u}+\epsilon \hat{u}_{m}\right) \frac{\partial v^{\prime}}{\partial x}+\epsilon \hat{v}_{m} \frac{\partial v^{\prime}}{\partial y}+\left(\frac{1}{\bar{\rho}}-\frac{\epsilon \hat{\rho}_{m}}{\bar{\rho}^{2}}\right) \frac{\partial p^{\prime}}{\partial y} \\
& -\mu \frac{1}{\bar{\rho}}\left(\frac{\partial^{2} v^{\prime}}{\partial x^{2}}+\frac{\partial^{2} v^{\prime}}{\partial y^{2}}+\frac{\partial^{2} v^{\prime}}{\partial z^{2}}\right) \\
& +\epsilon \frac{\partial \hat{v}_{m}}{\partial x} u^{\prime}+\epsilon \frac{\partial \hat{v}_{m}}{\partial y} v^{\prime}-\frac{\epsilon}{\gamma \bar{\rho} \bar{p}} \frac{\partial \hat{p}_{m}}{\partial y} p^{\prime}=0 \\
\frac{\partial w^{\prime}}{\partial t}+ & \left(\bar{u}+\epsilon \hat{u}_{m}\right) \frac{\partial w^{\prime}}{\partial x}+\epsilon \hat{v}_{m} \frac{\partial w^{\prime}}{\partial y}+\left(\frac{1}{\bar{\rho}}-\frac{\epsilon \hat{\rho}_{m}}{\bar{\rho}^{2}}\right) \frac{\partial p^{\prime}}{\partial z} \\
& -\mu \frac{1}{\bar{\rho}}\left(\frac{\partial^{2} w^{\prime}}{\partial x^{2}}+\frac{\partial^{2} w^{\prime}}{\partial y^{2}}+\frac{\partial^{2} w^{\prime}}{\partial z^{2}}\right)=0 \\
& +\epsilon \frac{\partial \hat{p}_{m}}{\partial x} u^{\prime}+\epsilon \frac{\partial \hat{p}_{m}}{\partial y} v^{\prime}+\epsilon \gamma\left(\frac{\partial \hat{u}_{m}}{\partial x}+\frac{\partial \hat{v}_{m}}{\partial y}\right) p^{\prime}=0 \\
\frac{\partial p^{\prime}}{\partial t}+ & \left(\bar{u}+\epsilon \hat{u}_{m}\right) \frac{\partial p^{\prime}}{\partial x}+\epsilon \hat{v}_{m} \frac{\partial p^{\prime}}{\partial y}+\gamma\left(\bar{p}+\epsilon \hat{p}_{m}\right) \\
& \times\left(\frac{\partial u^{\prime}}{\partial x}+\frac{\partial v^{\prime}}{\partial y}+\frac{\partial w^{\prime}}{\partial z}\right) \\
& \left.-\frac{\gamma-1}{C_{p}} \frac{\partial^{2} p^{\prime}}{\partial x^{2}}+\frac{\partial^{2} p^{\prime}}{\partial y^{2}}+\frac{\partial^{2} p^{\prime}}{\partial z^{2}}\right) \\
& +7)
\end{aligned}
$$

The boundary conditions are

$$
u^{\prime}=v^{\prime}=w^{\prime}=\frac{d p^{\prime}}{d y}=0 \quad \text { at } \quad y=H_{1},-H_{2} .
$$

The density $\rho^{\prime}$ will be determined by means of the locally isentropic approximation i.e. $\rho^{\prime}=\left(\bar{a}+\epsilon \hat{a}_{m}\right)^{-2} p^{\prime}$. In the above, $\mu, \kappa$, and $\gamma$ are the shear viscosity coefficient, thermal conductivity, and specific heat ratio, respectively. Here, $\mu$ and $\kappa$ are temperature dependent. At high-flow Mach number, and especially in the presence of large temperature gradients, such dependence must be taken into account. In this work, the principal objective is to demonstrate the existence of parametric instabilities induced by a periodic Mach wave system. For this purpose, a simpler, although less accurate, physical model in which $\mu$ and $\kappa$ are constants will be adopted. Such an approximate model offers great simplicity in the analysis and should be adequate for the limited objective of this paper. It is to be noted that, in the momentum equations above, the viscous terms involving the second derivatives in $x$ are found to be numerically insignificant at high Reynolds number. They may be neglected without incurring noticeable numerical errors. It is easy to find that the coefficients of (5)-(8) are periodic in $x$ and independent of $t$ and $z$. On following the Floquet theory (see Herber ${ }^{15}$ ), the parametric instability wave solution may be expressed in the form, 


$$
\begin{aligned}
{\left[\begin{array}{l}
u^{\prime} \\
v^{\prime} \\
w^{\prime} \\
p^{\prime}
\end{array}\right] } & =e^{i(\beta z-w)} e^{i v x} f(x, y) \\
& =e^{i(\beta z-\alpha)^{\prime}} e^{i v x} \sum_{t=-\infty}^{\infty}\left[\begin{array}{l}
u_{l}(y) \\
v_{l}(y) \\
w_{l}(y) \\
p_{l}(y)
\end{array}\right] e^{i l k_{i n} x} .
\end{aligned}
$$

In (9), $v$ is a characteristic exponent. When $v={ }_{2} k_{m}$, (9) gives the subharmonic solutions. When $\nu=0,(9)$ gives the fundamental solutions. When $v \neq 0$ or $\frac{1}{2} k_{m}$, the solution is detuned. Since $v$ and $v+l k_{m}$ yield identical expressions for any integer $l$, it is sufficient to assume that $0 \leqslant v<k_{m}$. For temporal instabilities that are considered in this paper, the value of $v$ is given.

Upon substituting (9) into (5)-(8) and equating terms of the same periods in $x$, an infinite system of ordinary differential equations in $y$ for the amplitude functions of expansion (9) is found. The exact form is given in Appendix A. For computational purposes, the systern will be truncated. Suppose the terms in (9) with $l=\ldots L_{1},-L_{1}+1, \ldots, L_{2}$ are to be retained, then the finite order ordinary differential equation system can be written in the following matrix form:

$$
\mathbf{E} \frac{d^{2} \mathbf{G}}{d y^{2}}+\mathbf{F} \frac{d \mathbf{G}}{d y}+\mathbf{C G}-(i \omega) \mathbf{G}=0
$$

where

$$
\mathbf{G}=\left[\begin{array}{cc}
u & -L_{1} \\
v & \iota_{1} \\
w & -L_{1} \\
p-L_{-1} \\
\vdots \\
u_{L_{2}} \\
v_{L_{2}} \\
w_{L_{2}} \\
p_{L_{2}}
\end{array}\right]_{4 M \times 1}=\left[\begin{array}{c}
g_{1}(y) \\
g_{2}(y) \\
\vdots \\
\vdots \\
g_{M_{s}}(y)
\end{array}\right]_{M_{s} \times 1} \cdot
$$

The boundary conditions for $\mathbf{G}$ are

$$
u_{t}=v_{i}=w_{i}=\frac{d p_{i}}{d y}=0, \quad y=-H_{2}, H_{1}
$$

for $l=-L_{1},-L_{1}+1, \ldots, L_{2}$.

It is easy to see that Eq. (10) and boundary conditions (11) form an eigenvalue problem. For temporal instabilities, the value of $v$ is given and $\omega$ and $\mathbf{G}$ are to be solved as eigenvalues and eigenfunctions.

\section{COMPUTATION METHODS}

\section{A. Spectral-collocation method}

To solve $(10)$, the spectral-collocation method ${ }^{18}$ will be used. This method converts the differential eigenvalue problem into an algebraic one.

Let $\mathbf{C}$ be approximated by polynomial expansions in the form

$$
\mathbf{G}=\left[\begin{array}{c}
\sum_{n=0}^{N} g_{1}^{(n)} \phi_{1}^{(n)}(y) \\
\sum_{n=0}^{N} g_{2}^{(n)} \phi_{2}^{(n)}(y) \\
\vdots \\
\vdots \\
\sum_{n=0}^{N} g_{M}^{(n)} \phi_{M_{s}}^{(n)}(y)
\end{array}\right] .
$$

Here, the basis polynomials $\phi_{j}^{(n)}(y)$ are chosen such that boundary conditions (11) are satisfied automatically. The exact forms of $\phi_{j}^{(n)}(y)$, in terms of Chebyshev polynomials, and the collocation points are given in Appendix $B$. The coefficients of the expansions $g_{j}^{(n)}$ are the unknowns. To ensure an adequate distribution of collocation points in the nondinensionalized physical space $[-1,+1](H / 2$ is the length scale), it is mapped into a computational space $[-1$, +1 J by a mapping function. This aspect is discussed in Appendix B. Now, upon substituting the spectral expansion (12) into the ordinary differential equation (10) and demanding that (10) be satisfied exactly at the collocation points at $y=y_{k}, k=0,1,2, \ldots, N$, an algebraic system of equations for the coefficients $g_{i}^{(n)}$ is found

$$
\begin{aligned}
& \sum_{j=1}^{M_{s}} \sum_{n=0}^{N}\left(e_{i j} \frac{d^{2}}{d y^{2}} \phi_{j}^{(n)}+f_{i j} \frac{d}{d y} \phi_{j}^{(n)}\right. \\
& \left.\quad+c_{i j} \phi_{j}^{(n)}-(i \omega) \phi_{j}^{(n)}\right)_{y=y_{k}} g_{j}^{(n)}=0
\end{aligned}
$$

for $i=1,2, \ldots, M_{5}, k=0,1,2, \ldots, N$, where $e_{i j}, f_{i j}$, and $c_{i j}$ are the elements of matrices $\mathbf{E}, \mathbf{F}$, and $\mathbf{C}$ of (10).

Equation (13) consists of $M_{s} \times(N+1)$ homogeneous algebraic equations for $M_{s} \times(N+1)$ coefficients $g_{j}^{(n)}$. It can easily be casted in the form of a generalized eigenvalue problem

$$
\mathrm{Ag}=(i \omega) \mathrm{Bg}
$$

where $\mathrm{g}$ is a vector containing the coefficients $g_{j}^{(n)}$ for $j=1$, $2, \ldots, M_{\xi}, n=0,1,2, \ldots, N$.

\section{B. The algebraic eigenvalue problem}

By carefully rearranging the rows and columns of (14) it is possible to put matrices $\mathbf{A}$ and $\mathbf{B}$ into block tridiagonal and block diagonal forms. Furthermore, since the boundary conditions are satisfied automatically by the basis polynomials, $B$ is nonsingular. The generalized eigenvalue problem may be put into the form of a standard algebraic eigenvalue problem

$$
\left[\begin{array}{cccccc}
\mathbf{A}_{11} & \mathbf{A}_{12} & 0 & 0 & \cdots & 0 \\
\mathbf{A}_{21} & \mathbf{A}_{22} & \mathbf{A}_{23} \ldots & 0 & \cdots & 0 \\
0 & \mathbf{A}_{32} & \mathbf{A}_{33} & \mathbf{A}_{34} & \cdots & 0 \\
\vdots & \cdots & \ddots & \ddots & \ddots & \vdots \\
\vdots & \ddots & \ddots & \ddots & \ddots & \mathbf{A}_{M-1 M} \\
0 & \cdots & \cdots & 0 & \mathbf{A}_{M M-1} & \mathbf{A}_{M M}
\end{array}\right] \mathbf{g}=\lambda \mathrm{g}
$$

where $\lambda=i \omega$ and $M$ is the number of Fourier modes retained in the truncated expansion. 
TABLE I. First 15 eigenvalues listed in the order of decreasing growth rate. $\epsilon=0.000, M=4, N=80$.

\begin{tabular}{rr}
\hline \hline No. & Eigenvalue $\omega H / \bar{u}$ \\
\hline 1 & $4.4261+0.227058 i$ \\
2 & $14.7788+0.171145 i$ \\
3 & $13.9597+0.148020 i$ \\
4 & $7.5891+0.102084 i$ \\
5 & $3.3474+0.088174 i$ \\
6 & $15.7039+0.077435 i$ \\
7 & $13.0052+0.059564 i$ \\
8 & $3.9231+0.052460 i$ \\
9 & $16.7104+0.020316 i$ \\
10 & $5.5544+0.019343 i$ \\
11 & $9.4649+0.006913 i$ \\
12 & $6.6076-0.002274 i$ \\
13 & $1.5515-0.002300 i$ \\
14 & $12.0282-0.004392 i$ \\
15 & $1.5169-0.005152 i$ \\
\hline
\end{tabular}

In principle, the above algebraic eigenvalue problem can be solved by standard solvers (e.g., EISPACK) using the $Q R$ algorithm, by which all the eigenvalues and eigenvectors are determined simultaneously. The $Q R$ algorithm, however, uses full storage for the matrices. Because of the limitation of computer central memory (even for the ETA10 supercomputer) the size of the matrix that can be handled by the $Q R$ algorithm is limited. For example, if the ETA10 supercomputer is used, the maximum size of the matrix cannot exceed 1000. Under these conditions, it is found that no more than six Fourier modes (each amplitude is approximated by 40 Chebyshev polynomials) can be included in the truncated Floquet solutions of (9). This is too small a number of Fourier modes to assure numerical convergence. To overcome this difficulty, the inverse iteration method ${ }^{19}$ is used to refine an approximate eigenvalue found by the $Q R$ algorithm. The inverse iteration method is an iterative scheme that finds an eigenvalue of the matrix that is closest to a given initial guess value. This method can be implemented such that only the nonzero block matrices of (15) are stored instead of the full

TABLE II. First 15 eigenvalues listed in the order of decreasing growth rate. $\epsilon=0.003, M=4, N=80$.

\begin{tabular}{rrr}
\hline \hline No. & Eigenvalue $\omega / H / \bar{u}$ & No. in Table I \\
\hline 1 & $4.4390+0.283532 i$ & 1 \\
2 & $12.7804+0.172778 i$ & 2 \\
3 & $7.6112+0.150544 i$ & 4 \\
4 & $13.9579+0.147166 i$ & 3 \\
5 & $15.7050+0.076094 i$ & 6 \\
6 & $13.0062+0.060194 i$ & 7 \\
7 & $1.9164+0.058523 i$ & new \\
8 & $3.8625+0.055412 i$ & 8 \\
9 & $3.4488+0.052387 i$ & 5 \\
10 & $5.5422+0.025431 i$ & 10 \\
11 & $16.7130+0.020519 i$ & 9 \\
12 & $9.4656+0.005610 i$ & 11 \\
13 & $1.5512-0.002438 i$ & 13 \\
14 & $6.6076-0.002991 i$ & 12 \\
15 & $3.7456-0.003059 i$ & 23 \\
\hline \hline
\end{tabular}

TABLE III. Numerical results showing convergence of the new instability wave solution, $\epsilon=0.003$.

\begin{tabular}{crrr}
\hline \hline $\begin{array}{c}\text { Approximation } \\
\text { sequence }\end{array}$ & $N$ & $\boldsymbol{M}$ & Eigenvalue $\omega H / \bar{u}_{1}$ \\
\hline 1 & 80 & 4 & $1.91638+0.0585234 i$ \\
2 & 80 & 8 & $1.91791+0.0563136 i$ \\
3 & 80 & 16 & $1.91806+0.0564042 i$ \\
4 & $100^{-}$ & 16 & $1.91803+0.0564178 i$ \\
\hline
\end{tabular}

matrix. ${ }^{20}$ This technique allows the eigenvalue calculation to process with much larger size matrices. In this work, the $Q R$ algorithm is used as an initial search of the eigenvalues of a smaller truncated matrix of the Fourier-Chebyshev expansion. Then the inverse iteration scheme is employed to refine the eigenvalues one at a time. During the eigenvalue refinement process, higher-order Fourier and Chebyshev terms are included.

\section{NUMERICAL RESULTS}

For the purpose of showing computationally that the presence of a period Mach wave can induce new parametric instability waves in a supersonic shear layer, the case $M_{1}=2.0, \quad M_{2}=1.2, \quad \bar{a}_{1} / \bar{a}_{2}=3.0, \quad \gamma_{1}=\gamma_{2}=1.4$, $H_{1}=H_{2}=H / 2, \quad \lambda_{m} / H=0.4, \quad \delta_{\omega} / H=0.05, \quad \beta=0$, $v=\frac{1}{2} k_{m}$, where $\delta_{\omega}$ is the vorticity thickness of the mixing layer is considered. In addition, the Reynolds number $\left(\bar{p}_{1} \bar{u}_{1} \delta_{\omega} / \mu\right)$ is taken to be 500 and the Prandtl number $\left(c_{p} \mu / \kappa\right)$ is assumed to be unity. To start the search for new instabilities, a truncated system consisting of 80 Chebyshev polynomials in the $y$ direction and four Fourier modes $(l=-2,-1,0,1)$ in the $x$ direction is used initially. This leads to an algebraic eigenvalue problem with a $972 \times 972$ matrix. This matrix is solved twice by the $Q R$ algorithm of the EISPACK subroutines. In the first run, $\epsilon$ is set equal to zero. This corresponds to the case with no. Mach waves. The first 15 eigenvalues of this run are listed in the order of decreasing growth rate in Table $\mathrm{I}$. There are 11 unstable eigenvalues. In the second run, $\epsilon$ is set equal to 0.003 corresponding to the presence of a weak Mach wave system. The first 15 eigenvalues are listed in Table II. There are now 12 unstable eigenvalues. By comparing the unstable eigenvalues of the two tables and accounting for some slight changes in the eigenvalues because of the interaction of the original instability waves with the Mach wave system, it is seen that eigen-

TABLE IV. Numerical results showing convergence of the new instability wave solution, $\epsilon=0.01$.

\begin{tabular}{crcc}
\hline $\begin{array}{c}\text { Approximation } \\
\text { sequence }\end{array}$ & $N$ & $M$ & Eigenvalue $\omega H / \bar{u}_{1}$ \\
\hline 1 & 80 & 16 & $2.06924+0.234844 i$ \\
2 & 80 & 20 & $2.06912+0.235120 i$ \\
3 & 80 & 24 & $2.06893+0.235171 i$ \\
4 & 100 & 20 & $2.07282+0.233876 i$ \\
5 & 100 & 28 & $2.07247+0.233796 i$ \\
\hline
\end{tabular}



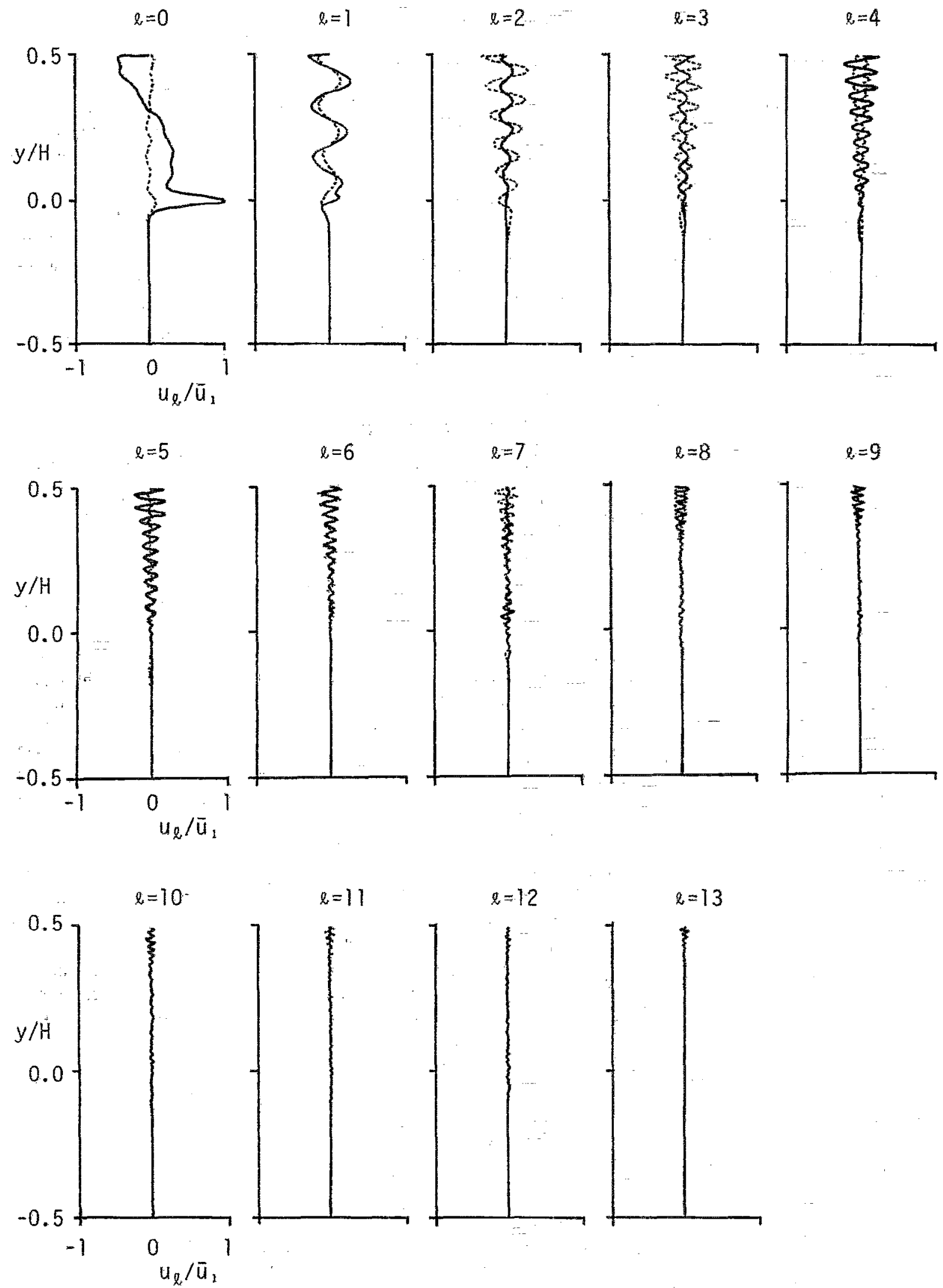

FIG. 2. Spatial distributions of different Fourier modes of the eigenfinction ( $u$ component) of the new instability wave. $l=$ order of Fourier mode; - $-{ }_{x}$ real parts: $\cdots \cdots$,imaginary parts. $\omega H / \bar{u}_{1}=(2.07247+0.233 .796 i), N=100, M=28, \epsilon=A_{0} / \lambda_{s t}=0.01 . M_{1}=2.0, M_{2}=1.2, \bar{a}_{1} / \bar{a}_{2}=3.0, \gamma_{1}=\gamma_{2}=1.4$, $\lambda_{m} / H=0.4, \delta_{m}^{-} / H=0.05, \beta=0, v=\frac{1}{2} k_{m}$. 

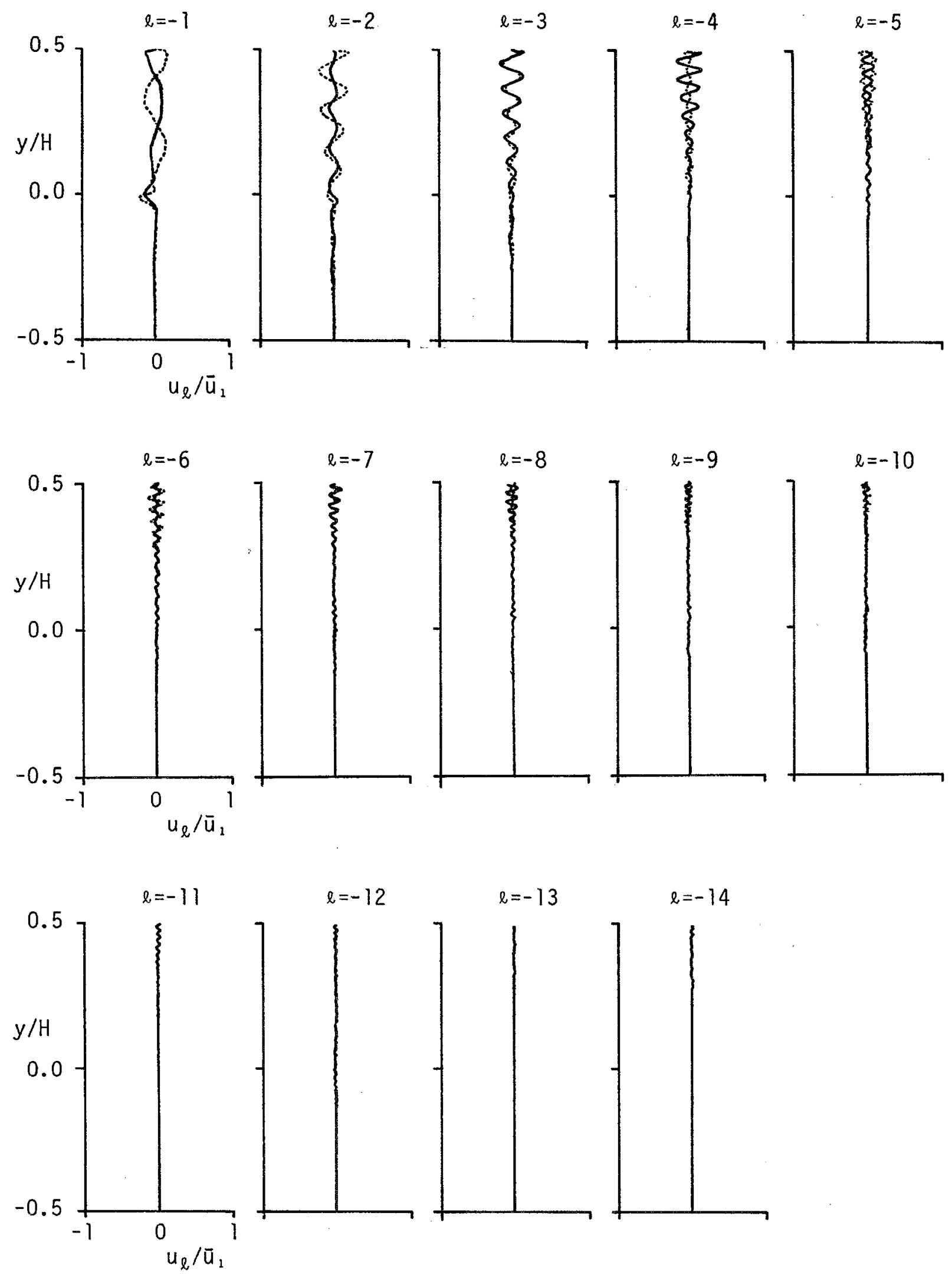

FIG. 2. (Continued.) 
$l=0$

$\ell=1$

$z=2$

$\ell=3$

$l=4$
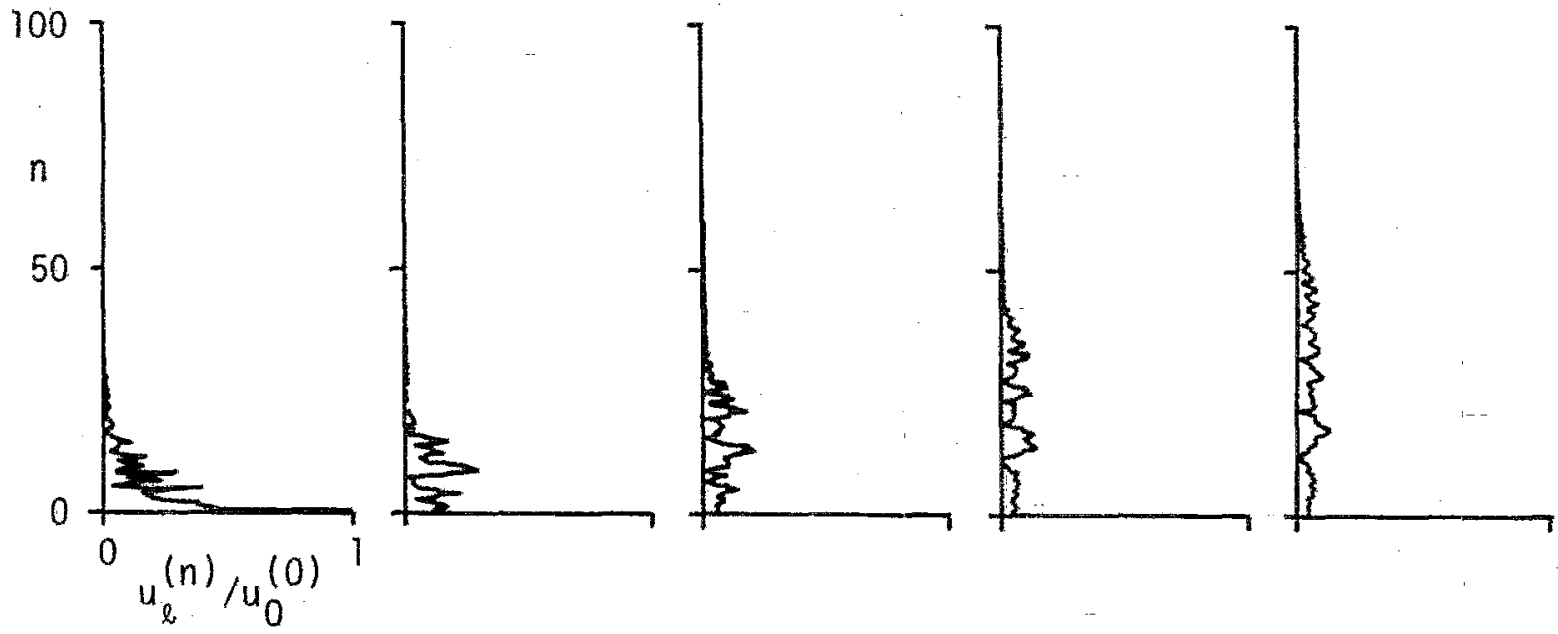

$Q=5$

$2=6$

$\ell=7$

$z=8$

$\ell=9$
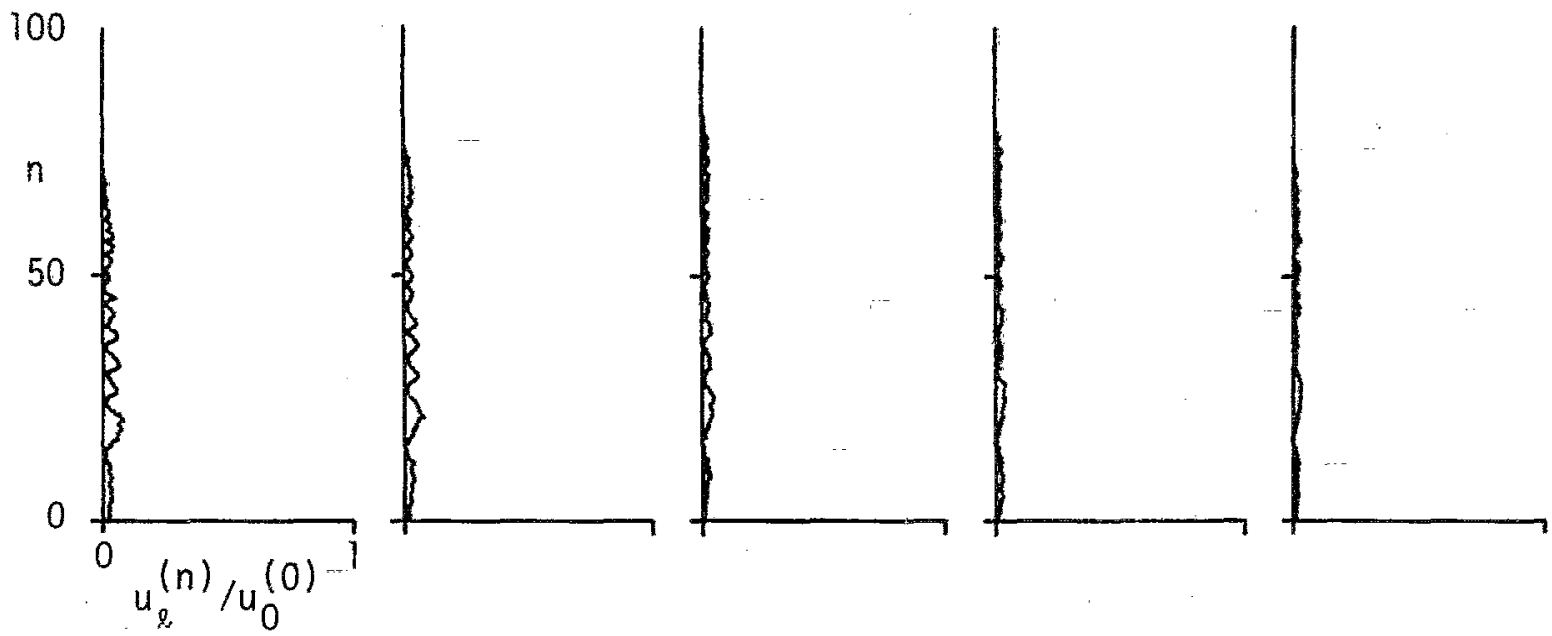

$l=10$

$b=11$

$x=12$

$a=13$.
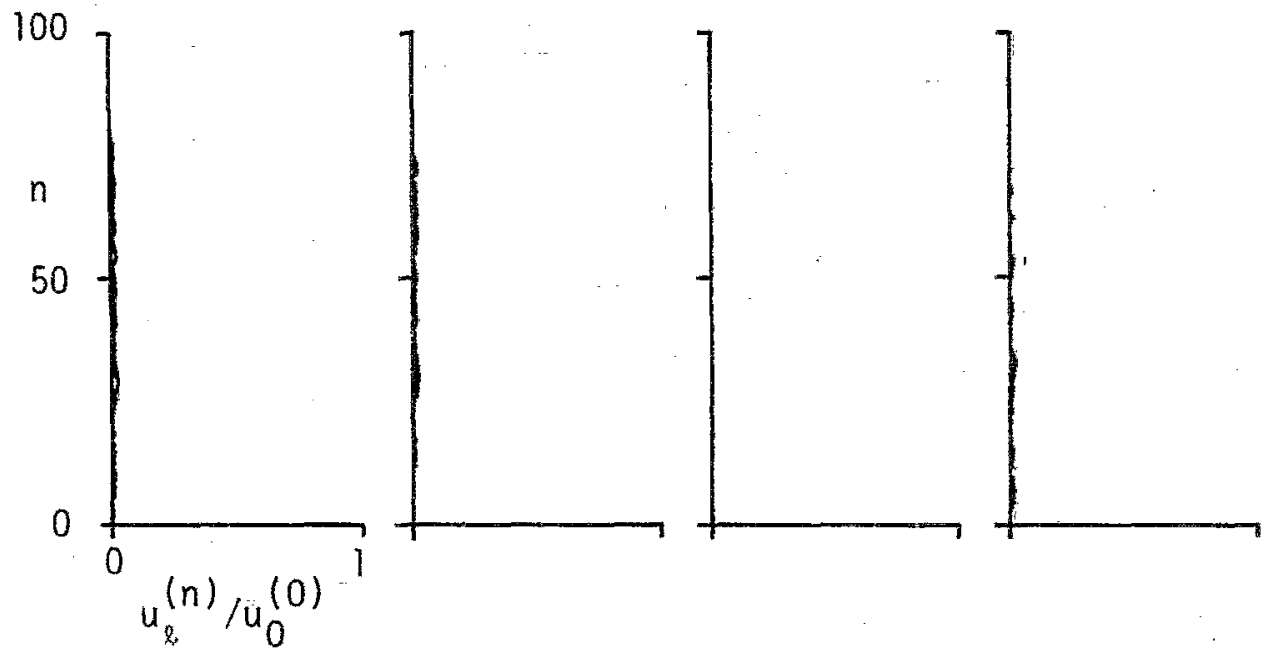

FIG. 3. Distribution of the relative magnitudes of the spectral coefficients of individual Fourier modes $(u$ component). $n=$ order of the basis function, $l=$ vider of Fourier component, $\omega H / \bar{u}_{1}=(2.07247+0.233796 i), N=100, M=28, \epsilon=A_{0} / \lambda_{m}=0.01, M_{1}=2.0, M_{2}=1.2, \bar{a}_{1} / \bar{a}_{2}=3.0, \gamma_{1}=\gamma_{2}=1.4_{2}$ $\lambda_{m} / H=0.4, \delta_{c} / H=0.05, \beta=0, v=1 k_{r n}$. 

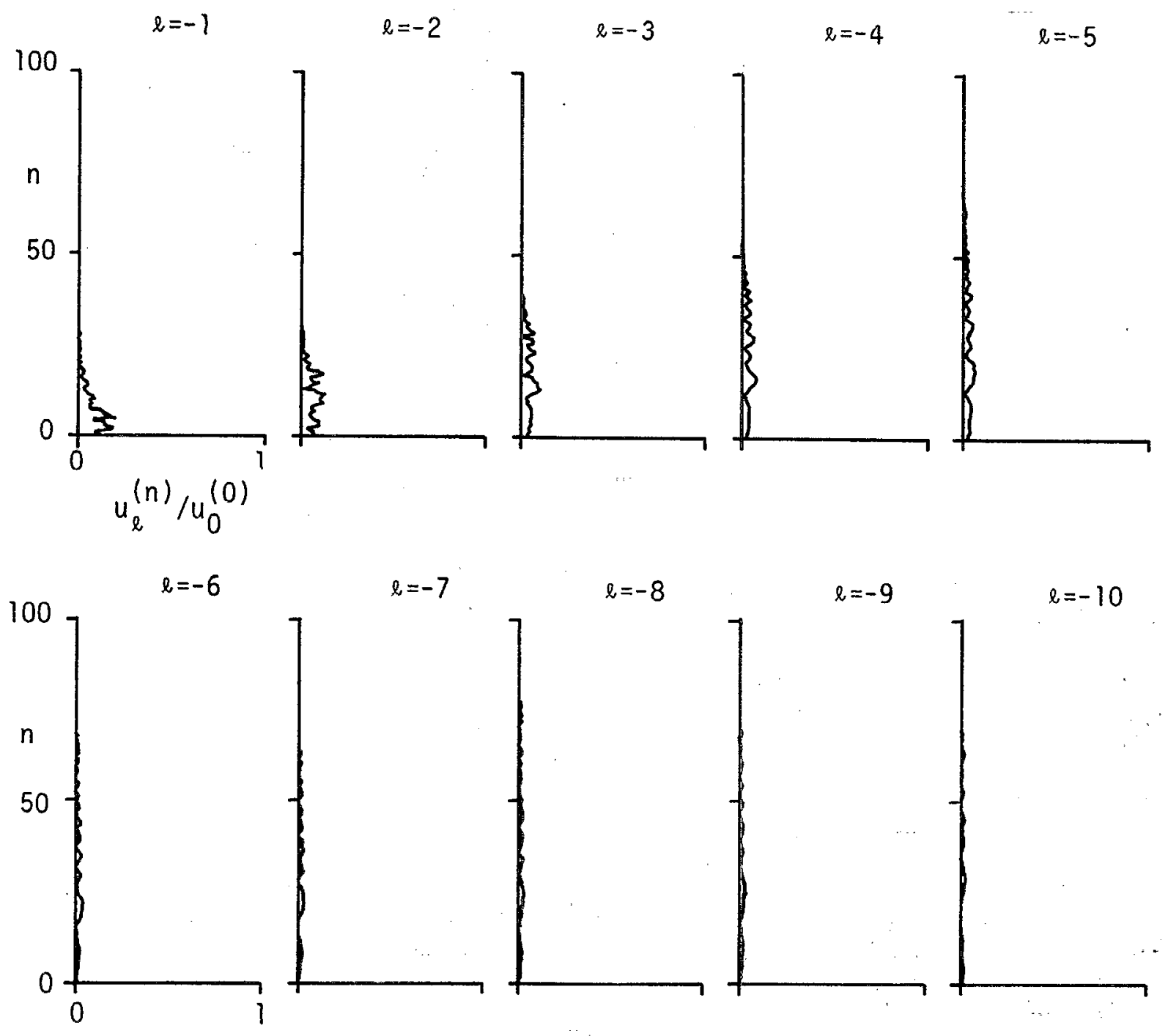

$$
u_{l}^{(n)} / u_{0}^{(0)}
$$
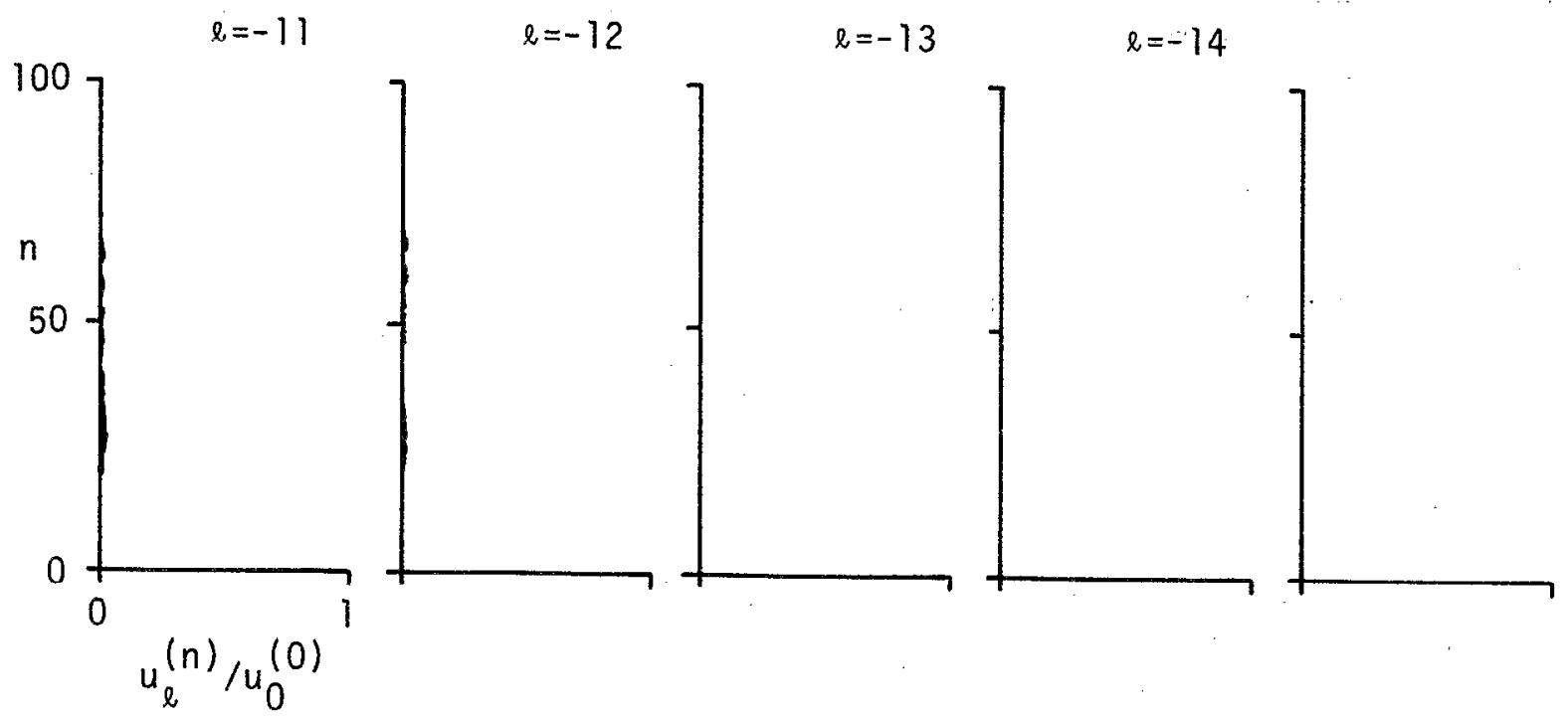

FIG. 3. (Continued.) 


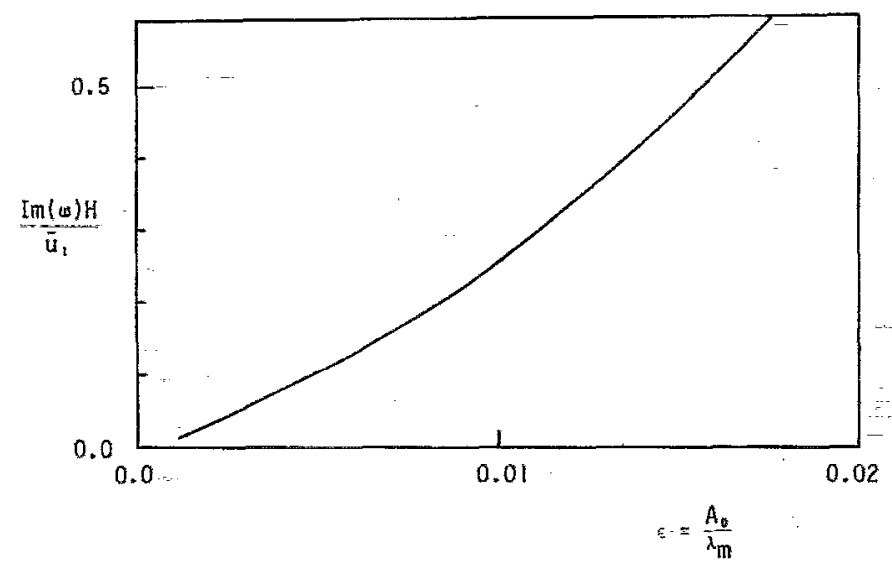

FIG. 4. Growth rate of the new instability wave as a function of wavy wall amplitude to wavelength ratio $A_{0} / \lambda_{m} . M_{1}=2.0, M_{2}=1.2, \bar{a}_{1} / \bar{a}_{2}=3.0$, $\gamma_{1}=\gamma_{2}=1.4, \lambda_{m} / H=0.4, \delta_{c o} / H=0.05$

value No. 7 in Table II is a possible candidate for new instability waves. In order to be sure that it is, indeed, a new parametric instability wave, it is necessary to demonstrate numerical convergence both in eigenvalue and eigenfunction as the number of terms in the Fourier-Chebyshev expansion increases.

The No. 7 eigenvalue in Table II is used as an initial guess value for eigenvalue refinement using the inverse iteration algorithm. ${ }^{19,20}$ In performing the inverse iteration process, the number of Fourier modes $(M)$ and Chebyshev basis polynomials $(N)$ are significantly increased. Table III provides the list of computed eigenvalues for different $M$ and $N$. In each calculation, the previous converged value is used as the starting value. From this table it is clear that the eigenvalue converges, at least, to four significant figures when $N=80, M=16$.

After having demonstrated numerical convergence for $\epsilon=0.003$, the inverse iteration eigenvalue refinement procedure is then applied to flows with successively stronger
Mach waves (i.e., larger $\epsilon$ ) using the previous eigenvalue as a starting value. Table IV shows the trend of convergence as $M$ and $N$ increase for $\epsilon=0.01$. Again, numerical convergence of the eigenvalue to four significant figures is realized at $N=100$ and $M=28$. Figure 2 shows the spatial distributions of the various Fourier modes that make up the eigenfunction. Clearly, the Fourier terms decrease in amplitude rapidly as the order increases, as those of a converged eigenfunction should. Figure 3 shows plots of the coefficients of the Chebyshev expansion of each Fourier mode. Again, the distributions of these coefficients are consistent with those of a converged eigenfunction.

The dependence of the growth rate of the new parametric instability wave on the strength of the Mach wave system is given in Fig. 4. For $\epsilon<0.01$, the dependence is nearly linear. The fact that the growth rate increases monotonically with the strength of the Mach waves further indicates that the existence of this new, instability wave is tied to the presence of the Mach wave system. At $\epsilon=0.015$, the growth rate of the new instability wave is larger than the maximum growth rate of the instability waves of the supersonic shear layer without Mach waves given in Table I. Thus it appears that the suggestion of inducing instability waves of significant growth rate by means of a periodic Mach wave system to enhance mixing in a supersonic shear layer may, indeed, be possible.

\section{ACKNOWLEDGEMENTS}

This work was supported in part by the Office of Naval Research under Grant No. N00014-87-J-1130 and also in part by the Florida State University through time granted on its ETA 10 and Cray YMP supercomputers.

\section{APPENDIX A: EQUATIONS FOR THE AMPLITUDE FUNCTIONS}

The system of ordinary differential equations for $u_{l}, v_{l}, w_{l}$, and $p_{l}$ obtained by substituting (9) into $(5)-(8)$ is

$$
\begin{aligned}
& -i \omega\left[\begin{array}{c}
u_{l} \\
v_{l} \\
w_{l} \\
p_{l}
\end{array}\right]+\left\{i\left(v+l k_{m}\right)\left[\begin{array}{cccc}
\bar{u} & 0 & 0 & \frac{1}{\bar{\rho}} \\
0 & \bar{u} & 0 & 0 \\
0 & 0 & \bar{u} & 0 \\
\bar{p} & 0 & 0 & \bar{u}
\end{array}\right]+i \beta\left[\begin{array}{cccc}
0 & 0 & 0 & 0 \\
0 & 0 & 0 & 0 \\
0 & 0 & 0 & \frac{1}{\bar{\rho}} \\
0 & 0 & \bar{p} & 0
\end{array}\right]+\left[\begin{array}{cccc}
0 & \frac{d \bar{u}}{d y} & 0 & 0 \\
0 & 0 & 0 & 0 \\
0 & 0 & 0 & 0 \\
0 & 0 & 0 & 0
\end{array}\right]\right\} \\
& {\left[\begin{array}{l}
u_{l} \\
v_{l} \\
w_{l} \\
p_{l}
\end{array}\right]+\left[\begin{array}{cccc}
0 & 0 & 0 & 0 \\
0 & 0 & 0 & \frac{1}{\bar{\rho}} \\
0 & 0 & 0 & 0 \\
0 & \bar{p} & 0 & 0
\end{array}\right] \frac{d}{d y}\left[\begin{array}{c}
u_{l} \\
v_{l} \\
w_{l} \\
p_{l}
\end{array}\right]-\frac{\mu}{\bar{\rho}}\left[\begin{array}{ccccc}
1 & 0 & 0 & 0 \\
0 & 1 & 0 & 0 \\
0 & 0 & 1 & 0 \\
0 & 0 & 0 & \gamma & 0 \\
-1
\end{array}\right]-\frac{d^{2}}{d y^{2}}\left[\begin{array}{c}
u_{l} \\
v_{l} \\
w_{l} \\
p_{l}
\end{array}\right]} \\
& +\left[\left(\nu+l k_{m}\right)^{2}+\beta^{2}\right] \frac{\mu}{\bar{\rho}}\left[\begin{array}{cccc}
1 & 0 & 0 & 0 \\
0 & 1 & 0 & 0 \\
0 & 0 & 1 & 0 \\
0 & 0 & 0 & \gamma-1
\end{array}\right]\left[\begin{array}{c}
u_{l} \\
v_{l} \\
w_{l} \\
p_{l}
\end{array}\right]
\end{aligned}
$$




$$
\begin{aligned}
& +\epsilon\left\{i\left[v+(l-1) k_{m}\right]\left[\begin{array}{cccc}
\hat{u}_{+} & 0 & 0 & -\frac{\hat{\rho}_{+}}{\bar{\rho}^{2}} \\
0 & \hat{u}_{+} & 0 & 0 \\
0 & 0 & \hat{u}_{+} & 0 \\
\gamma \hat{p}_{+} & 0 & 0 & \hat{u}_{+}
\end{array}\right]+i \beta\left[\begin{array}{cccc}
0 & 0 & 0 & 0 \\
0 & 0 & 0 & 0 \\
0 & 0 & 0 & -\frac{\hat{\rho}_{+}}{\bar{\rho}^{2}} \\
0 & 0 & \gamma \hat{p}_{+} & 0
\end{array}\right]\right. \\
& \left.+\left[\begin{array}{cccc}
i k_{m} \hat{u}_{+} & \frac{d \hat{u}_{+}}{d y} & 0 & -\frac{i k_{m} \hat{p}_{+}}{\gamma \bar{p} \bar{p}} \\
i k_{m} \hat{u}_{+} & \frac{d \hat{v}_{+}}{d y} & 0 & -\frac{1}{\gamma \bar{\rho} \bar{p}} \frac{d \hat{p}_{+}}{d y} \\
0 & 0 & 0 & 0 \\
i k_{m} \hat{p}_{+} & \frac{d \hat{p}_{+}}{d y} & 0 & \gamma\left(i k_{m} \hat{u}_{+}+\frac{d \hat{v}_{+}}{d y}\right)
\end{array}\right]\right\}\left[\begin{array}{c}
u_{l-1} \\
v_{l-1} \\
w_{l-1} \\
p_{l-1}
\end{array}\right] \\
& +\epsilon\left\{i\left[v+(l+1) k_{m}\right]\left[\begin{array}{cccc}
\hat{u}_{-} & 0 & 0 & -\frac{\hat{\rho}_{-}}{\bar{\rho}^{2}} \\
0 & \hat{u}_{--} & 0 & 0 \\
0 & 0 & \hat{u}_{-} & 0 \\
\gamma \hat{p}_{-} & 0 & 0 & \hat{u}_{-}
\end{array}\right]+i \beta\left[\begin{array}{cccc}
0 & 0 & 0 & 0 \\
0 & 0 & 0 & 0 \\
0 & 0 & 0 & -\frac{\hat{\rho}_{-}}{\bar{\rho}^{2}} \\
0 & 0 & \gamma \hat{p}_{-} & 0
\end{array}\right]\right. \\
& \left.+\left[\begin{array}{cccc}
i k_{m} \hat{u}_{-} & \frac{d \hat{u}_{-}}{d y} & 0 & \frac{i k_{m} \hat{p}_{-}}{\gamma \hat{\rho} \bar{p}} \\
i k_{m} \hat{u}_{-} & \frac{d \hat{v}_{-}}{d y} & 0 & -\frac{1}{\gamma \bar{p}} \frac{d \hat{p}_{-}}{d y} \\
0 & 0 & 0 & 0 \\
i k_{m} \hat{p}_{-} & \frac{d \hat{p}_{-}}{d y} & 0 & \gamma\left(i k_{m} \hat{u}_{-}+\frac{d \hat{v}_{-.}}{d y}\right)
\end{array}\right]\right\}\left[\begin{array}{c}
u_{l+1} \\
v_{l+1} \\
w_{l+1} \\
p_{l+1}
\end{array}\right]+\epsilon\left[\begin{array}{cccc}
\hat{v}_{+} & 0 & 0 & 0 \\
0 & \hat{v}_{+} & 0 & -\frac{\hat{\rho}_{+}}{\bar{\rho}^{2}} \\
0 & 0 & \hat{v}_{+} & 0 \\
0 & 0 & \gamma \hat{p}_{+} & \hat{v}_{+}
\end{array}\right] \frac{d}{d y}\left[\begin{array}{c}
u_{l-1} \\
v_{l-1} \\
w_{l-1} \\
p_{l-1}
\end{array}\right] \\
& +\epsilon\left[\begin{array}{cccc}
\hat{v}_{-} & 0 & 0 & 0 \\
0 & \hat{v}_{-} & 0 & -\frac{\hat{\rho}_{-}}{\bar{\rho}^{2}} \\
0 & 0 & \hat{v}_{-} & 0 \\
0 & 0 & \gamma \hat{p}_{-} & \hat{v}_{-}
\end{array}\right] \cdot \frac{d}{d y}\left[\begin{array}{c}
u_{l+1} \\
v_{l+1} \\
w_{l+1} \\
p_{l+1}
\end{array}\right]=0, \quad \text { for } l=0, \pm 1, \pm 2, \ldots .
\end{aligned}
$$

\section{APPENDIX B: BASIS AND MAPPING FUNCTIONS}

Chebyshev polynomials $T_{n}(y)$ are used to form the basis functions for the spectral expansion (9). In order to satisfy the boundary conditions at the end points of the normalized interval [ $-1,1]$ (using $H / 2$ as the length scale), each polynomial is combined either with $T_{0}(y)$ or $T_{1}(y)$ depending on whether $n$ is even or odd to form the basis polynomials.

(a) For velocity components $u, v$, and $w$, the following basis polynomials are used:

$$
\begin{aligned}
\Phi^{(n)} & = \begin{cases}T_{0}(y)-T_{n+2}(y), & n \text { even, } \\
T_{1}(y)-T_{n+2}(y), & n \text { odd },\end{cases} \\
& \Rightarrow \Phi^{(n)}( \pm 1)=0 .
\end{aligned}
$$

(b) For pressure $p$, the basis polynomials $\Psi_{(n)}$ are used:

$$
\Psi^{(n)}(y)= \begin{cases}1, & n=0, \\ \left(\frac{n+2}{2}\right)^{2} T_{2}(y)-T_{n+2}(y), & n \text { even, } \\ (n+2)^{2} T_{1}(y)-T_{n+2}(y), & n \text { odd, }\end{cases}
$$

$$
\Rightarrow \frac{d \Psi^{(n)}}{d y}( \pm 1)=0
$$

The collocation points are the zeros of $T_{N+1}(y)$, i.e.,

$$
y_{k}=\cos \left(\frac{(2 k+1) \pi}{2 N+2}\right), \quad k=0,1,2, \ldots, N .
$$

It is easy to see that the collocation points are dense around the end points of the interval $[-1,1]$. In order to place more points in the center region where the mixing layer is, the following coordinate transformation (mapping) from $y \in[-1,1]$ to the computational coordinate $Y \in[-1,1]$ is used:

$$
\begin{aligned}
y= & \frac{1}{2}\left[\left(\frac{1}{3}+\frac{9}{7}\right) Y+0.1\left(\frac{9}{7}-\frac{1}{3}\right)\right. \\
& \left.\times \ln \left(\frac{\cosh [(|Y|-0.3) / 0.1]}{\cosh (0.3 / 0.1)}\right) \operatorname{sign}(Y)\right] .
\end{aligned}
$$


'H. Ikawa and T. Kubota, AIAA I. 13,566 (1975).

${ }^{2}$ D. Papamoschou and A. Roshko, AIAA Paper No. 86-0162, 1986.

${ }^{3}$ D. Papamoschou and A. Roshko, I. Fluid Mech. 197, 453 (1988).

${ }^{4}$ N. Chinzei, G. Masuya, T. Komuro, A. Murakami, and K. Kudou, Phys.

Fluids 29, 1345 (1986).

5. A. Ragab and J. L. Wu, AIAA Paper No. 88-0038, 1988

'S. A. Ragab and J. L. Wu, Phys, Fluíds A 1, 957 (1989).

${ }^{7}$ T. L. Jackson and C. E. Grosch, J. Fluid Mech. 208, 609 (1989).

s. T. L. Jackson and C. E. Grosch, Phys. Fluids A 2, 949 (1990).

${ }^{9} \mathrm{C} . \mathrm{K}$. W. Tam and F. Q. Hu, AIAA Paper No. $88-3675,1988$.

${ }^{10} \mathrm{C} . \mathrm{K}$. W. Tam and F. Q. Hu, J. Fluid Mech. 203, 51 (1989).

${ }^{1}$ J. Greenough, J. Riley, M. Soetrisno, and D. Eberhardt, AIAA Paper No. 89-0372, 1989.
${ }^{12}$ M. Zhuang, T. Kubota, and P. E. Dimotakis, Phys. Fluids A 2, 599 (1990).

${ }^{13}$ C. K. W. Tam and F. Q. IIu, Bull. Am. Phys. Soc. 34, 2285 (1989).

${ }^{14}$ C. K. W. Tam and F. Q. Hu, to appear in J. Fluid Mech. (1991).

${ }^{15}$ T. Herbert, Annu. Rev. Fluid Mech. 20, 487 (1988).

ins. A. Orszag and A. T. Patera, J. Fluid Mech. 128, 347 (1983).

${ }^{17} \mathrm{H}$. W. Liepmann and A. Roshko, Elements of Gasdynamics (Wiley, New York, 1957).

${ }^{18} \mathrm{~J}$. P. Boyd, Mon. Weather Rev. 106, 1192 (1978).

${ }^{19} \mathrm{~J}$. H. Wilkinson, The Algebraic Eigenvalue Problem (Oxford U. $\mathrm{P}_{,} \mathrm{Ox}-$ ford, 1965).

${ }^{20}$ W. Kerner, in Large Scaĩe Eigênvalue Problems, edited by J. Cullen and R. A. Willough (North-Holland, Amsterdam; 1986). 